EPJ manuscript No.

(will be inserted by the editor)

\title{
Longitudinally Polarized Photoproduction of Inclusive Hadrons at Fixed-Target Experiments
}

\author{
B. Jäger ${ }^{1}$, M. Stratmann ${ }^{2}$, and W. Vogelsang ${ }^{34}$ \\ 1 Institut für Theoretische Physik, Universität Karlsruhe, D-76128 Karlsruhe, Germany \\ ' 2 Institut für Theoretische Physik, Universität Regensburg, D-93040 Regensburg, Germany \\ 3 Physics Department, Brookhaven National Laboratory, Upton, New York 11973, U.S.A. \\ 4 RIKEN-BNL Research Center, Brookhaven National Laboratory, Upton, New York 11973 - 5000, U.S.A.
}

\begin{abstract}
We present a detailed phenomenological study of spin-dependent single-inclusive high- $p_{T}$ hadron photoproduction with particular emphasis on the kinematics relevant for the CompAss and HERMES fixedtarget experiments. We carefully examine the theoretical uncertainties associated with the only moderate transverse momenta accessible in such measurements and analyze the sensitivity of the relevant spin asymmetries to the gluon polarization in the nucleon as well as to the completely unknown parton content of circularly polarized photons.
\end{abstract}

PACS. 13.88. $+\mathrm{e}-12.38 . \mathrm{Bx}-13.85 . \mathrm{Ni}$

\section{Motivation and Introduction}

To measure the so far largely unknown polarization of gluons, $\Delta g$, in the nucleon is a key goal of several current experiments in high-energy nuclear physics. The successful start of polarized proton-proton collisions at the BNL Relativistic Heavy Ion Collider (RHIC) has opened up new, unequaled possibilities [1. Gluon polarization can be accessed in a variety of high transverse momentum processes such as single-inclusive hadron, jet, prompt photon, or heavy quark production. Compass [2] at CERN and HERMES [3] at DESY, instead, scatter beams of longitudinally polarized leptons $l$ off polarized fixed targets $N$. Here, in particular high- $p_{T}$ hadron pairs, both in photoproduction and in deep-inelastic electroproduction, have been identified by the experiments to provide a promising tool to gain some knowledge about $\Delta g[4]$.

A meaningful extraction of parton densities from experiment requires a reliable interpretation of the underlying data in the framework of perturbative QCD (pQCD). Factorization theorems [5] assure that in the presence of a large scale, for instance, a high transverse momentum $p_{T}$, a cross section can be written as a convolution of partonic hard scattering cross sections, which depend on the process under consideration, certain combinations of nonperturbative, universal parton densities, and, if applicable, fragmentation functions. The standard pQCD framework receives corrections which are suppressed by inverse powers of the large scale characterizing the process. Since it is not a priori clear where these "power corrections" become relevant, the factorized ansatz has to be scrutinized for each measurement. For all spin-dependent observables, cross sections or spin asymmetries, this is best achieved by studying first the unpolarized cross sections because both ingredients, the parton densities and the hard scattering cross sections, are known very well here. Only if a reasonable agreement between theory and experiment is established within their respective uncertainties can one have confidence that a similar measurement with polarization can be used to extract information about the spin structure of the nucleon.

First measurements at RHIC of unpolarized high- $p_{T}$ neutral pion [6] and prompt photon [7] production rates turned out to be in good agreement with theoretical predictions [8, 10] even down to unexpectedly small values of the transverse momentum of about $1.5 \mathrm{GeV}$. For this comparison it is essential to compute the perturbatively calculable short-distance cross sections up to the nextto-leading order (NLO) approximation of pQCD. On the one hand, NLO corrections turn out to be sizable, on the other hand, theoretical uncertainties related to the renormalization and factorization procedures are much reduced compared to lowest order (LO) estimates. Therefore much effort was put in calculating the NLO pQCD corrections also for spin-dependent processes in recent years.

The PHENIX collaboration at RHIC has recently released first, partly preliminary, results for high- $p_{T}$ neutral pion production with longitudinally polarized protons [11. However, from this measurement alone it is difficult and way too early to draw any conclusions about the gluon polarization $\Delta g$ in the nucleon; see also [12. To map out $\Delta g(x, \mu)$ over a sufficiently large range in the momentum fraction $x$, which is ultimately required for a determination of the first moment of $\Delta g$, and scale $\mu$ (to test the scale evolution predicted by pQCD) a much better statis- 


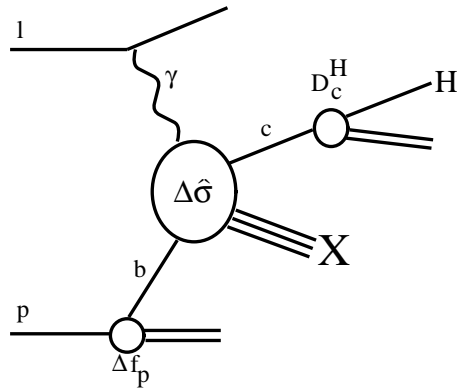

(a)

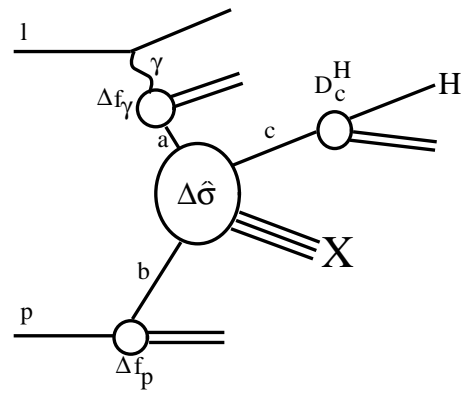

(b)

Fig. 1. Generic direct (a) and resolved (b) photon contributions to the photoproduction of a hadron $H$.

tical accuracy and a significantly larger coverage in $p_{T}$ are needed. As a rule of thumb, a measurement of a final state jet or hadron with a certain $p_{T}$ predominantly probes the gluon density at momentum fractions $x \gtrsim 2 p_{T} / \sqrt{S}$ where $\sqrt{S}$ is the available proton-proton center-of-mass system (c.m.s.) energy. Since the cross section drops rapidly with increasing $p_{T}$ it will take RHIC quite some time to accumulate enough events to determine $\Delta g$ at $x \gtrsim 0.1$. This is where the fixed-target experiments at much lower energies can possibly help to add information about $\Delta g$ at medium-to-large $x$ in the very near future.

In the case of hadron-pair production, $l N \rightarrow l^{\prime} H_{1} H_{2} X$, the process already considered by COMPAss and HERMES 4], the theoretical framework is rather complex and so far NLO corrections are still lacking. In this paper we therefore focus on the photoproduction of single-inclusive hadrons, $l N \rightarrow l^{\prime} H X$, where the complete NLO pQCD framework is already at hand [8,13] and has been recently applied to study the physics case of a conceivable first polarized $e p$-collider at BNL 14. Photoproduction with quasi-real photons has the advantage of much higher rates than deeply-inelastic electroproduction of hadrons. The price to pay is the more involved theoretical framework for photoproduction where so-called "direct" and "resolved" photons contribute to the cross section [15] as depicted in Figs. 1 (a) and (b), respectively. Estimates for the latter require knowledge of the parton content of circularly polarized photons which is lacking at the moment. We will demonstrate below that this does not, however, seriously limit the usefulness of this process.

We note that there are actually already some photoproduction data for double-longitudinal spin asymmetries in $e N \rightarrow H^{ \pm} X$ from the E155 experiment at SLAC [16]. Here the scattered electron was not observed, so the data set is integrated over all virtualities of the exchanged photon. Nonetheless, thanks to the photon propagator, production by almost real photons strongly dominates. The E155 data have been compared to the theoretical predictions of [17, which were based on LO calculations supplemented by certain higher-twist contributions. A relatively poor overall agreement was found. To achieve a better description of the data, the theoretical framework used in 17. was subsequently augmented by estimates [18] of soft contributions that are mostly relevant at small trans- verse momenta. We will later present a brief comparison of our NLO calculations with the E155 data.

At fixed-target energies it is much less clear that pQCD methods are applicable than at collider energies. In fact, data for high- $p_{T}$ processes in hadron-hadron fixed-target scattering have in the past been a serious challenge for the factorized framework outlined above 19. It is therefore even more important to demonstrate first that unpolarized production rates can be described with standard pQCD methods before turning to studies of spin asymmetries. Otherwise conclusions about the parton and/or spin content of nucleons might be misleading or wrong. We will therefore not only present results for spin asymmetries and discuss their possible sensitivity to $\Delta g$ but also focus on predictions for unpolarized reference or "benchmark" cross sections which allow to probe the pQCD framework.

The paper is organized as follows: in Sec. 2 we briefly recall the theoretical framework for photoproduction required for the subsequent numerical sections and set up our notation. We refrain from repeating lengthy technical details and refer the interested reader to Refs. [8,13. We then turn to a detailed study of single-inclusive pion production in the kinematical regions relevant for COMPAss and HeRMEs including, as far as possible, experimental cuts. Detailed phenomenological results are presented in Secs. 3.1 and 3.2 for CompAss and HERMES, respectively. Special emphasis is put on theoretical uncertainties associated with the application of perturbative methods in the domain of the rather moderate transverse momenta accessible in fixed-target experiments. We first present unpolarized "benchmark" cross sections for each experiment and then turn to a discussion of the relevant spin asymmetries and their sensitivity to $\Delta g$. We comment on the relevance of resolved photon contributions and their influence on the extraction of the gluon polarization. In Sec. 3.3 we present the comparison to the E155 data. We conclude in Sec. 4

\section{Technical Prerequisites}

In the following, we consider the spin-dependent photoproduction cross section $l N \rightarrow l^{\prime} H X$ for the scattering of a longitudinally polarized lepton beam $l$ off a target nucleon $N$ with subsequent hadronization of a produced parton $c$ into the observed hadron $H$ with momentum $P_{H}$, see 
Fig. 11 The observed hadron $H$ is at high transverse momentum $p_{T}$, ensuring the required large momentum transfer. The differential single-inclusive cross section can then be schematically written as a convolution

$$
\begin{aligned}
& d \Delta \sigma \equiv \frac{1}{2}\left[d \sigma_{++}-d \sigma_{+-}\right] \\
& =\sum_{a, b, c} \int d x_{a} d x_{b} d z_{c} \Delta f_{a}^{l}\left(x_{a}, \mu_{f}\right) \Delta f_{b}^{N}\left(x_{b}, \mu_{f}\right) \\
& \times d \Delta \hat{\sigma}_{a b \rightarrow c X}\left(S, x_{a}, x_{b}, P_{H} / z_{c}, \mu_{r}, \mu_{f}, \mu_{f}^{\prime}\right) D_{c}^{H}\left(z_{c}, \mu_{f}^{\prime}\right) .
\end{aligned}
$$

The subscripts "++" and "+-" in Eq. (1) denote the helicities of the colliding leptons and nucleons.

In Eq. (2) $x_{b}$ is the fraction of the nucleon momentum taken by parton $b$, and the $\Delta f_{b}^{N}\left(x_{b}, \mu_{f}\right)$ are the usual polarized parton densities. For instance, the spin-dependent gluon distribution at a scale $\mu_{f}$ is defined by

$$
\Delta g\left(x_{b}, \mu_{f}\right) \equiv g_{+}\left(x_{b}, \mu_{f}\right)-g_{-}\left(x_{b}, \mu_{f}\right),
$$

where the subscripts \pm indicate the helicity of a gluon in a nucleon $N$ of positive helicity. The non-perturbative functions $D_{c}^{H}\left(z_{c}, \mu_{f}^{\prime}\right)$ describe the fragmentation of parton $c$ $(c=q, \bar{q}, g)$ into the observed hadron $H$ at a momentum scale $\mu_{f}^{\prime} . z_{c}$ is the fraction of parton $c$ 's momentum taken by the hadron $H$. The sum in Eq. (2) runs over all partonic channels $a+b \rightarrow c+X$ contributing to the singleinclusive cross section $l N \rightarrow l^{\prime} H X$ with $d \Delta \hat{\sigma}_{a b \rightarrow c X}$ the associated spin-dependent partonic hard scattering cross sections. The latter can be calculated in pQCD order-byorder in the strong coupling constant $\alpha_{s}\left(\mu_{r}\right)$, with $\mu_{r}$ denoting the renormalization scale, and are known up to NLO accuracy. The spin-dependent results can be found in 8,1320 . The experimentally measured cross section is the sum of the so-called "direct" and "resolved" photon contributions, cf. Figs. 1 (a) and (b), respectively,

$$
d \Delta \sigma=d \Delta \sigma_{\mathrm{dir}}+d \Delta \sigma_{\mathrm{res}}
$$

which both can be cast into the form of Eqs. (11) and (2) by defining $\Delta f_{a}^{l}$ appropriately. Most generally, the parton densities of the lepton $l, \Delta f_{a}^{l}$, can be written as convolutions,

$$
\Delta f_{a}\left(x_{a}, \mu_{f}\right)=\int_{x_{a}}^{1} \frac{d y}{y} \Delta P_{\gamma l}(y) \Delta f_{a}^{\gamma}\left(x_{\gamma}=\frac{x_{a}}{y}, \mu_{f}\right),
$$

with

$$
\begin{aligned}
\Delta P_{\gamma l}(y) & =\frac{\alpha_{e}}{2 \pi}\left\{\left[\frac{1-(1-y)^{2}}{y}\right] \ln \frac{Q_{\max }^{2}(1-y)}{m_{l}^{2} y^{2}}\right. \\
& \left.+2 m_{l}^{2} y^{2}\left(\frac{1}{Q_{\max }^{2}}-\frac{1-y}{m_{l}^{2} y^{2}}\right)\right\}
\end{aligned}
$$

being the spin-dependent Weizsäcker-Williams "equivalent photon" spectrum [21] that describes the collinear emission of a photon with low virtuality $Q^{2}$ less than some upper limit $Q_{\max }^{2}$ (determined by the experimental conditions) by a lepton of mass $m_{l}$.

The explicit form of the $\Delta f_{a}^{\gamma}$ in Eq. (5) depends on the specifics of the interaction that the quasi-real photon radiated off the lepton undergoes in the hard scattering. For the direct photon contribution to the cross section, $d \Delta \sigma_{\text {dir }}$, depicted in Fig. 1 (a), parton $a$ in Eq. (2) has to be identified with an elementary photon and hence $x_{a}$ with the momentum fraction $y$ of the photon w.r.t. the parent lepton, i.e.,

$$
\Delta f_{a}^{\gamma}=\delta\left(1-x_{\gamma}\right)
$$

in Eq. (5). If the photon first resolves into hadronic "constituents" which then interact with partons of the target nucleon $N, \Delta f_{a}^{\gamma}$ in Eq. (5) represents the parton densities of a circularly polarized photon. The latter are defined in complete analogy to the ones for a nucleon target in Eq. (3). Unlike hadronic parton distributions which are genuinely non-perturbative objects, photonic densities consist of a perturbatively calculable "pointlike" contribution which dominates their behavior at large momentum fractions $x_{\gamma}$ in Eq. (5). This will become important in the discussion of the numerical results in the remainder of the paper.

We shall note that neither $d \Delta \sigma_{\text {dir }}$ nor $d \Delta \sigma_{\text {res }}$ individually are measurable cross sections as their definition depends on the specific details of the factorization procedure for dealing with singular collinear parton emissions. Only their sum, Eq. (4), is a physically meaningful quantity provided both $d \Delta \sigma_{\text {dir }}$ and $d \Delta \sigma_{\text {res }}$ have been calculated consistently in the same factorization scheme. Needless to say, the corresponding spin-averaged cross sections are straightforwardly obtained by replacing all polarized parton densities, partonic cross sections, etc., by their appropriate unpolarized counterparts.

Finally, we wish to stress that Eqs. (11) and (2) apply to a single-inclusive cross section, which is not to be confused with a cross section for producing a so-called "leading hadron". The latter, for which only the hadron with the highest momentum in the event is counted, is sometimes provided by experiment. It is not possible in the framework of $\mathrm{pQCD}$ based on the factorization theorem and outlined above to compute a "leading-hadron" cross section, as fragmentation functions $D_{c}^{H}$ are fully inclusive.

\section{Phenomenological Results}

With the technical framework and notation set up we are now in a position to perform a detailed phenomenological analysis of polarized and unpolarized single-inclusive pion photoproduction cross sections for the COMPASs and Hermes fixed-target experiments in Secs. 3.1 and 3.2 respectively. In Sec. 3.3 we present the comparison to the E155 data.

All our results will be differential in the hadron's transverse momentum $p_{T}$ and integrated over all kinematically and experimentally allowed pseudo-rapidities $\eta$ of the produced hadron $H$ measured w.r.t. the direction of the incident lepton beam. For the unpolarized parton densities of 
the nucleon and photon we adopt throughout the CTEQ 22] and GRV [23] sets, respectively. To study the sensitivity to the unknown gluon polarization of the nucleon we use four different sets of spin-dependent parton distributions emerging from the GRSV analysis [24]. These sets span a rather large range of gluon densities $\Delta g$ all consistent with present DIS data. Apart from the "standard" set of GRSV with a moderately large, positive $\Delta g$, the three other sets " $\Delta g=g$ input", " $\Delta g=0$ input", and " $\Delta g=-g$ input" are characterized by a large positive, a vanishing, and a large negative gluon polarization, respectively, at the input scale of the evolution.

To model the hadronization into pions, we use the fragmentation functions of KKP 25] which are known to provide a good description of the $p p \rightarrow \pi^{0} X$ cross sections measured by Phenix and Star [6] (and, of course, all available $e^{+} e^{-}$data). Although the framework outlined in Sec. 2 applies in principle to the photoproduction of any hadron species (made of light quark flavors only), we limit ourselves mainly to the production of neutral pions. These are most abundant, and the $\pi^{0}$ fragmentation functions, mainly extracted at large resolution scales $\mu \simeq M_{Z}$ from LEP data, have been shown to work quite well also at scales of a few $\mathrm{GeV}$ relevant here [26].

Since the parton distributions of circularly polarized photons, $\Delta f^{\gamma}$, required for estimates of the resolved photon contribution $d \Delta \sigma_{\text {res }}$, are completely unknown so far one has to resort to some models. For our purposes it is sufficient to use the two extreme scenarios proposed in Ref. 28 which are based on "maximal" $\left[\Delta f^{\gamma}\left(x, \mu_{0}\right)=\right.$ $\left.f^{\gamma}\left(x, \mu_{0}\right)\right]$ and "minimal" $\left[\Delta f^{\gamma}\left(x, \mu_{0}\right)=0\right]$ saturation of the positivity condition $\left|\Delta f^{\gamma}\left(x, \mu_{0}\right)\right| \leq f^{\gamma}\left(x, \mu_{0}\right)$ at the starting scale $\mu_{0}$ for the evolution to $\mu>\mu_{0}$. Both models result in very different parton distributions $\Delta f^{\gamma}$ at smallto-medium $x_{\gamma}$ while they almost coincide as $x_{\gamma} \rightarrow 1$ due to the dominance of the perturbatively calculable pointlike contribution in this region. Unless we study the impact of the details of the non-perturbative input to the evolution of $\Delta f^{\gamma}$ on the the full photoproduction cross section, the use of the "maximal" set will be implicitly understood. For recent work on the parton distributions of polarized photons, see [27.

\subsection{Single-Inclusive Pion Production at COMPASS}

With the present setup, CoMPASs scatters polarized muons off the deuterons in a polarized ${ }^{6} \mathrm{LiD}$ solid-state target. The beam energy is $E_{\mu}=160 \mathrm{GeV}$, corresponding to a lepton-nucleon c.m.s. energy of $\sqrt{S} \simeq 18 \mathrm{GeV}$. On average the beam polarization is $\mathcal{P}_{\mu} \simeq 76 \%$. About $\mathcal{F}_{d} \simeq 50 \%$ ("dilution factor") of the deuterons can be polarized, with an average polarization of $\mathcal{P}_{d} \simeq 50 \%$ 2. It is also conceivable to run in the future with a "proton target" $\left(\mathrm{NH}_{3}\right)$ which, however, will have a much less advantageous dilution factor of about $\mathcal{F}_{p} \simeq 17.6 \%$ but a polarization of $\mathcal{P}_{p} \simeq 85 \%$. We will therefore mainly present results for the photoproduction of neutral pions off a deuteron target.

Currently, pions can be detected if their scattering angle is less than $\theta_{\max }=70 \mathrm{mrad}$. Using $\eta=-\ln \tan (\theta / 2)$,

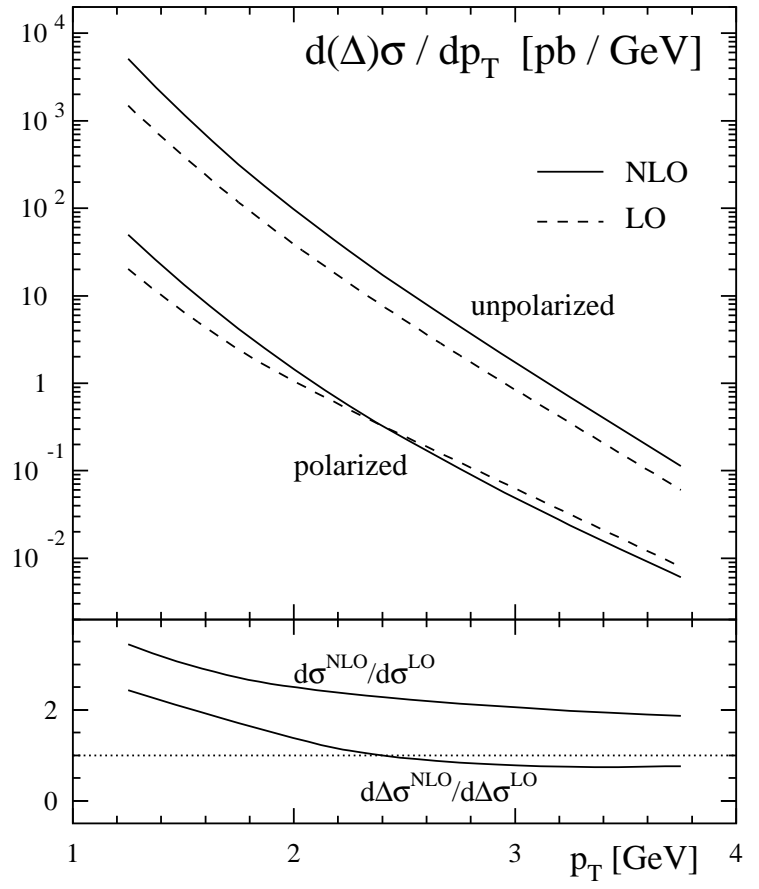

Fig. 2. Unpolarized and polarized $p_{T}$-differential singleinclusive cross sections at LO (dashed) and NLO (solid) for the photoproduction of neutral pions, $\mu d \rightarrow \mu^{\prime} \pi^{0} X$ at $\sqrt{S}=$ $18 \mathrm{GeV}$, integrated over the angular acceptance of Compass. The lower panel shows the ratios of NLO to LO contributions ( $K$-factor $)$.

this straightforwardly translates into a minimal bound on pseudo-rapidity $\eta^{\text {min }} \simeq 3.35$ in the laboratory frame, corresponding to $\eta_{c m s}^{\min } \simeq 0.44$ in the lepton-nucleon c.m.s. where we have made use of the well-known behavior of rapidity under Lorentz boosts:

$$
\eta_{c m s}=\eta-\frac{1}{2} \ln \frac{2 E_{\mu}}{M_{N}}
$$

with $M_{N}$ the nucleon mass. Kinematics sets an additional upper bound on the pion's rapidity depending on its transverse momentum: $\eta_{c m s}^{\max }=\cosh ^{-1}\left(\sqrt{S} / 2 p_{T}\right)$. As already mentioned, we will always integrate over all kinematically allowed rapidities, $0.44 \lesssim \eta_{c m s} \lesssim \cosh ^{-1}\left(\sqrt{S} / 2 p_{T}\right)$.

The momentum distribution of the quasi-real photons radiated off the muons can be described by the Weizsäcker-Williams spectrum given in Eq. (6) with $m_{l}=m_{\mu}$ and $Q_{\max }^{2}=0.5 \mathrm{GeV}^{2}$. The photon's momentum fraction $y$ is restricted to be in the range $0.2 \leq y \leq 0.9$. At smaller $y$ the photon polarization is strongly diluted as the unpolarized equivalent photon spectrum behaves like $\left(1+(1-y)^{2}\right) / y$ rather than $\left(1-(1-y)^{2}\right) / y$ in Eq. (6). We note that the often omitted non-logarithmic pieces in Eq. (6) result in a small but non-negligible contribution here.

Figure 2 shows our predictions for the $p_{T}$-differential cross section for the polarized and unpolarized photopro- 


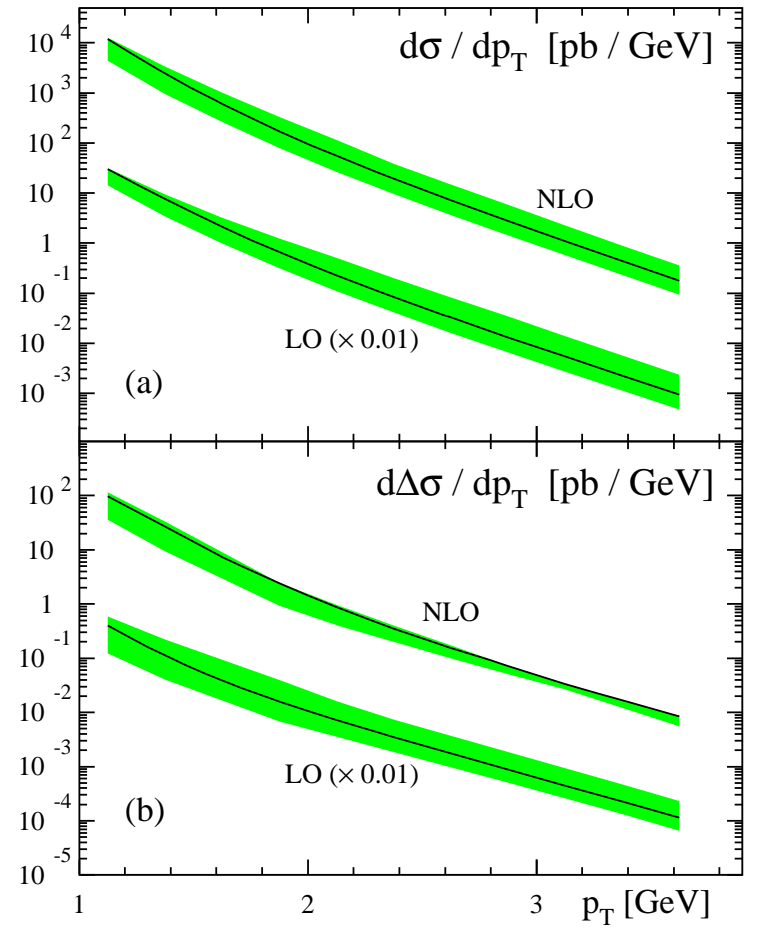

Fig. 3. Scale dependence of the LO and NLO unpolarized (a) and polarized (b) cross sections for $\mu d \rightarrow \mu^{\prime} \pi^{0} X$ shown in Fig. 2 All scales are varied simultaneously in the range $p_{T} / 2 \leq \mu_{r}=\mu_{f}=\mu_{f}^{\prime} \leq 2 p_{T}$. Solid lines correspond to the choice where all scales are set to $p_{T}$. All LO computations have been rescaled by a factor 0.1 to better distinguish them from the NLO results.

duction of neutral pions, $\mu d \rightarrow \mu^{\prime} \pi^{0} X$, at LO and NLO accuracy at COMPASS. We have set all factorization and renormalization scales in Eq. (2) equal to the pion transverse momentum, $\mu_{f}=\mu_{f}^{\prime}=\mu_{r}=p_{T}$. The so-called $K$-factor, defined as the ratio of NLO to LO unpolarized (polarized) cross sections,

$$
K=\frac{d(\Delta) \sigma^{\mathrm{NLO}}}{d(\Delta) \sigma^{\mathrm{LO}}}
$$

is depicted in the lower panel of Fig. 22 The results indicate clearly the relevance of the NLO corrections to the cross section in the small-to-medium $p_{T}$-region accessible at a fixed-target experiment, in particular in the unpolarized case. The effects of higher order corrections are less pronounced for the longitudinally polarized cross section, mainly due to large cancellations among the various partonic channels contributing to $d \Delta \sigma$ at NLO. The different behavior of the unpolarized and polarized $K$-factors also clearly indicates that the contributions due to NLO corrections do not cancel in the experimentally relevant double-spin asymmetry defined as

$$
A_{\mathrm{LL}}^{H, N} \equiv \frac{d \Delta \sigma}{d \sigma}=\frac{d \sigma_{++}-d \sigma_{+-}}{d \sigma_{++}+d \sigma_{+-}} .
$$

Not unexpectedly, at $p_{T} \simeq 1 \div 2 \mathrm{GeV}$, the $K$-factors for both, polarized and unpolarized cross sections rise sharply, perhaps indicating a breakdown of the standard pQCD framework as outlined in Sec. 2 Since, as will be demonstrated below, this is precisely the $p_{T}$-region where the statistical accuracy of COMPASS would best allow to deduce some information about $\Delta g$ from a measurement of the double-spin asymmetry $A_{\mathrm{LL}}^{\pi, d}$, one has to ensure the validity of the $\mathrm{pQCD}$ framework first. As already emphasized in the Introduction, this is best achieved by a measurement of the unpolarized cross section shown in Fig. 2] where all ingredients, partonic cross sections, parton distributions, and fragmentation functions are known. We note that all-order resummations of large logarithms in the perturbative series which appear when the initial partons have just enough energy to produce a high- $p_{T}$ pion and a recoiling massless "jet" may lead to a considerable enhancement of the cross section at fixed-target energies as was recently demonstrated for the process $p p \rightarrow \pi X$ 29. Similar calculations for the case of photoproduction are not yet available but certainly desirable. Any residual shortfall of the resummed theoretical prediction would then indicate the relevance of non-perturbative contributions.

Large $K$-factors, as found in Fig. 2 are, however, of limited significance for unambiguously estimating the impact of higher order corrections in a perturbative calculation. This is due to the large scale uncertainties associated with the LO cross sections entering the denominator of Eq. (9). We therefore further explore the reliability of the perturbative approach by studying the dependence of the calculated cross sections, Eq. (2), on the unphysical, a priori arbitrary factorization and renormalization scales, $\mu_{f}$, $\mu_{f}^{\prime}$ and $\mu_{r}$, respectively. Any dependence on these scales is a remnant of the truncation of the perturbation series at some fixed order of $\alpha_{s}$ and thus expected to diminish if higher order corrections are included. This is the prime motivation for going beyond the LO approximation of pQCD. The scales are of the order of the hard scale characterizing the process, here, the large $p_{T}$ of the observed hadron, but not further specified by theory. An estimate for the sensitivity of the computed cross section to $\mu_{f}, \mu_{f}^{\prime}$, and $\mu_{r}$ is usually obtained by varying them collectively in the range $p_{T} / 2 \leq \mu_{f}=\mu_{f}^{\prime}=\mu_{r} \leq 2 p_{T}$. We note that in principle all scales can be varied independently.

The shaded bands in Figs. B (a) and (b) indicate the resulting scale uncertainty of the unpolarized and polarized cross sections, respectively, shown in Fig. 22. In contrast to similar studies for inclusive high- $p_{T}$ pion hadro- 8] and photoproduction [13] at collider energies, where the theoretical scale uncertainties are substantially reduced when the NLO corrections are taken into account, this barely happens here. This is particularly true for the unpolarized cross section, whereas the scale dependence of the polarized cross section improves beyond the LO, but only slightly in the region $1 \leq p_{T} \leq 2 \mathrm{GeV}$ which mainly matters for a determination of $\Delta g$. Together with the large $K$-factors found in Fig. 2 this underlines the delicacy of a perturbative calculation in the low-energy range associ- 


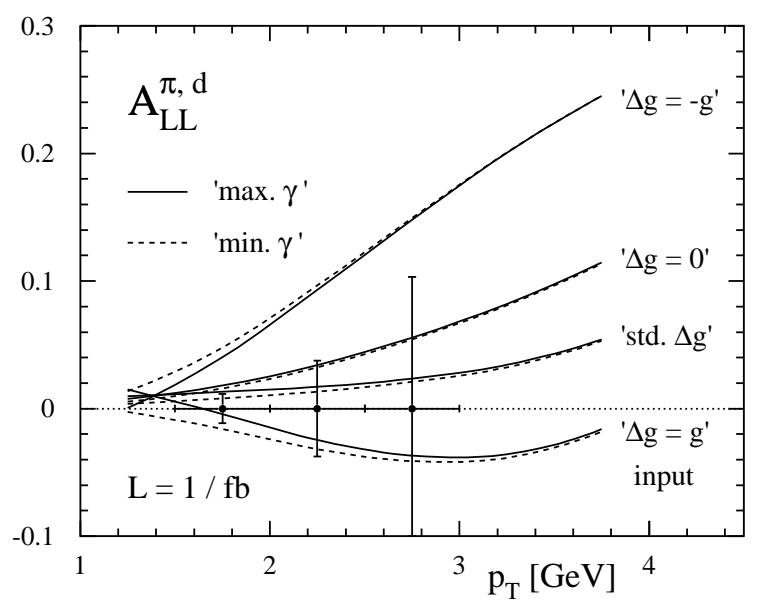

Fig. 4. Double-spin asymmetry $A_{\mathrm{LL}}^{\pi, d}$ at NLO for different gluon polarizations in the nucleon and minimal and maximal saturation of the polarized photon densities, dashed and solid lines, respectively (see text). The "error bars" indicate the estimated statistical uncertainty for such a measurement at COMPASS.

ated with fixed-target experiments such as Compass. It is therefore particularly important to check the applicability of pQCD methods by showing, for instance, that data taken in unpolarized collisions fall within the uncertainty band shown in Fig. 3

Next we consider the double spin asymmetry $A_{\mathrm{LL}}^{\pi, d}$, Eq. (10), for single-inclusive neutral pion production which will be one of the main quantities of interest in experiment. Fig. 团shows $A_{\mathrm{LL}}^{\pi, d}$, calculated at NLO for the "standard" set of the GRSV spin-dependent parton densities [24], as well as for three other sets emerging from the GRSV analysis which mainly differ in the assumptions about $\Delta g$ (see above). The impact of the unknown non-perturbative parton structure of the circularly polarized photon on $A_{\mathrm{LL}}^{\pi, d}$ is examined by making use of the two extreme sets also introduced at the beginning of Sec. 3. We refrain from showing LO estimates for the double-spin asymmetry which are of rather limited use anyway. Due to the pronounced differences in the $K$-factors for the unpolarized and polarized cross sections, see Fig. 2 the LO results for $A_{\mathrm{LL}}^{\pi, d}$ are considerably larger than the NLO ones shown in Fig. 4 This is in contrast to the frequently made assumption that NLO corrections cancel in spin asymmetries. We note that similar observations have been also made for hadroproduction of pions [8].

As can be seen, the actual choice of photonic parton densities barely affects the results for the spin asymmetry shown in Fig. 固 if the pion's transverse momentum is larger than about $2 \mathrm{GeV}$. This can be readily understood by noticing that in this region the average momentum fraction $\left\langle x_{a}\right\rangle$ in Eq. (15) is larger than 0.5, i.e., one probes only $x_{\gamma}$-values where the photon structure is dominated by the "pointlike" contribution independent of the details of the unknown non-perturbative input 28. In this $p_{T^{-}}$ region a measurement of the spin asymmetry could be related to a certain $\Delta g$. However, this does not imply that the resolved contribution to the cross section is negligible as we shall demonstrate below. On the other hand, for $p_{T} \lesssim 1.5 \mathrm{GeV}$ results for different gluon polarizations of the nucleon and assumptions about $\Delta f^{\gamma}$ overlap, making it virtually impossible to draw any conclusions without either knowing $\Delta g$ or $\Delta f^{\gamma}$.

To judge whether the observed dependence of $A_{\mathrm{LL}}^{\pi, d}$ on

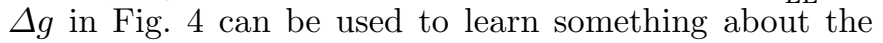
gluon polarization, we give estimates for the expected statistical accuracy $\delta A_{\mathrm{LL}}^{\pi, d}$ for such a measurement at ComPASS in certain bins of $p_{T}$, calculated from

$$
\delta A_{\mathrm{LL}}^{H, N} \simeq \frac{1}{\mathcal{P}_{\mu} \mathcal{P}_{N} \mathcal{F}_{N}} \frac{1}{\sqrt{\sigma_{b i n} \mathcal{L}}} .
$$

Here, $\sigma_{b i n}$ denotes the unpolarized cross section in the $p_{T^{-}}$ bin considered, $\mathcal{L}$ the integrated luminosity for which we assume $1 \mathrm{fb}^{-1}$, and all other parameters are as specified at the beginning of Sec. 3.1 Not unexpectedly, the statistical accuracy rapidly deteriorates towards higher $p_{T}$ as the photoproduction cross section drops sharply. In the region $1.5 \lesssim p_{T} \lesssim 2.5 \mathrm{GeV}$ it is conceivable that some information about $\Delta g$ can be obtained. We note that the gluon polarization will be predominantly probed in the range $0.1 \lesssim x_{b} \lesssim 0.3$ of the momentum fraction $x_{b}$ in Eq. (2).

Let us now turn to a closer analysis of how the results presented in Figs. 2 and 4 come about and can be understood, by studying the different contributions to the polarized photoproduction cross section separately. This is done in Fig. [5 Here we use again our default sets of parton densities: the "standard" set of GRSV 24] for the nucleon and the "maximal" set of 28] for the photon. First of all, Fig. [5 (a) reveals that the resolved photon cross section $d \Delta \sigma_{\text {res }}$ dominates over the direct contribution $d \Delta \sigma_{\text {dir }}$ in the entire range of $p_{T}$ relevant for Compass. At a first glance this result is counter-intuitive and requires further clarification since at fixed-target energies one might expect a dominance of the direct contributions to the full photoproduction cross section. The importance of the resolved photon contribution, in particular in the polarized case, can be readily understood by a closer inspection of the individual partonic subprocesses contributing to the full photoproduction cross section.

Figure 5(b) shows that indeed both direct channels, $\gamma q$ and $\gamma g$ scattering, exceed in magnitude any of the resolved subprocesses. However, the two channels contribute with different sign and therefore cancel each other to a large extent in the sum $d \Delta \sigma_{\mathrm{dir}}$. In this way numerically smaller resolved subprocesses can become important, in particular, as they all contribute with the same sign for a positive gluon polarization. We note that the partonic spin asymmetry $d \Delta \hat{\sigma} / d \hat{\sigma}$ for the LO process $\gamma g \rightarrow q \bar{q}$ is -1 , such that only positive $\Delta g$ lead to this cancellation pattern. Nevertheless, also for a large negative gluon polarization [" $\Delta g=-g$ input" in Fig. [5] (b)] is the resolved contribution non-negligible. For all relevant values of $p_{T}$ the bulk of the resolved contribution stems from the $q g$ subprocess where a quark at large momentum fraction $x_{\gamma}$ from the 


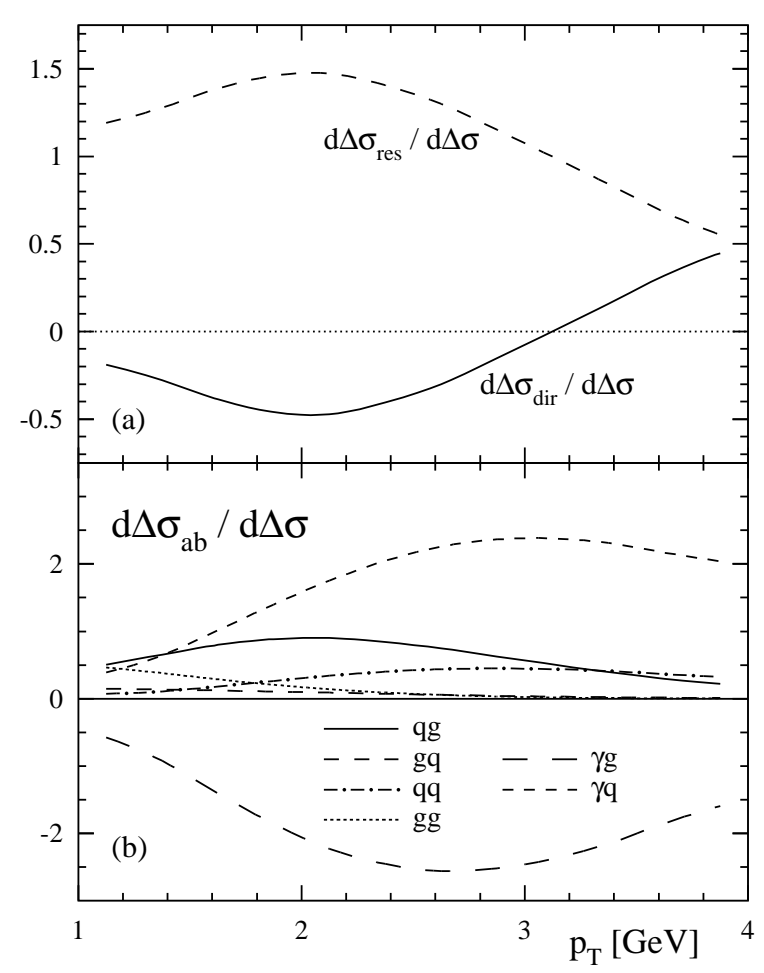

Fig. 5. (a) Direct and resolved $\mathrm{NLO}(\overline{\mathrm{MS}})$ contributions and (b) $\mathrm{NLO}(\overline{\mathrm{MS}})$ contributions of different partonic channels $a+$ $b \rightarrow c+X$ to the full NLO polarized photoproduction cross section shown in Fig. 2

photon scatters off a gluon from the nucleon [solid line in Fig. [5 (b)]. This is because quark densities in the photon are sizable at large $x_{\gamma}[23,28$ due to the pointlike contribution, contrary to parton densities in the nucleon which rapidly vanish as $x \rightarrow 1$. As mentioned before, at large momentum fractions $\Delta q^{\gamma}$ is not sensitive to the details of how the non-perturbative hadronic content of the photon was modeled. We therefore conclude that photoproduction of inclusive hadrons with COMPAss kinematics and statistics will most likely not yield much information on the non-perturbative nature of the resolved photon. On the other hand, this finding certainly simplifies an analysis of photoproduction data with $p_{T} \gtrsim 1.5 \mathrm{GeV}$ in terms of $\Delta g$ despite our ignorance of the photonic parton densities $\Delta f^{\gamma}$.

We obtain very similar results when considering a proton target, as will be realized when the CoMPAss experiment switches to a $\mathrm{NH}_{3}$ target in the future. The resulting spin asymmetry, $A_{\mathrm{LL}}^{\pi, p}$, is depicted in Fig. 6] To estimate the statistical accuracy we have again assumed an integrated luminosity of $1 \mathrm{fb}^{-1}$, and all other parameters are as specified at the beginning of Sec. 3.1 The sensitivity to $\Delta g$ and the rather weak dependence on the photon scenario for $p_{T} \gtrsim 1.5 \mathrm{GeV}$, characteristic for the large $x_{\gamma^{-}}$ region probed at COMPASS, are essentially the same as for $A_{\mathrm{LL}}^{\pi, d}$. We note that the intricate interplay between direct and resolved photon processes is quite similar to the one

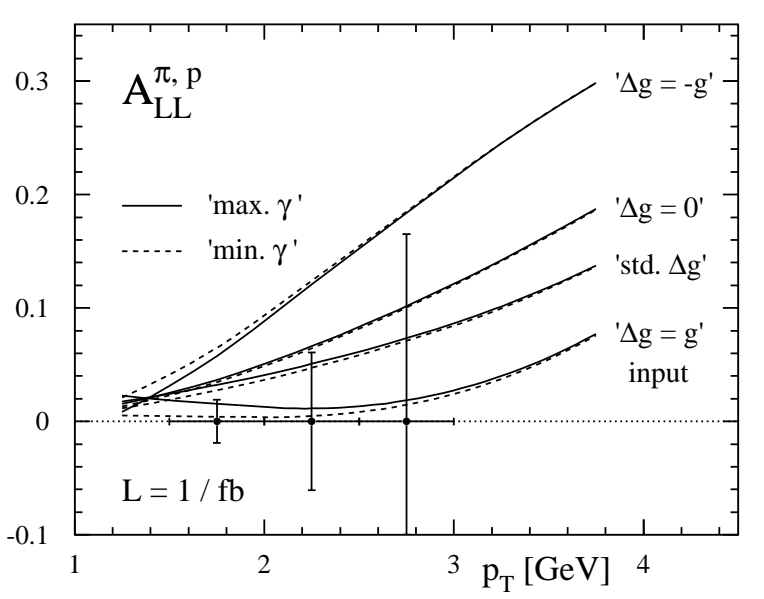

Fig. 6. As in Fig. 4 but now for a "proton" target.

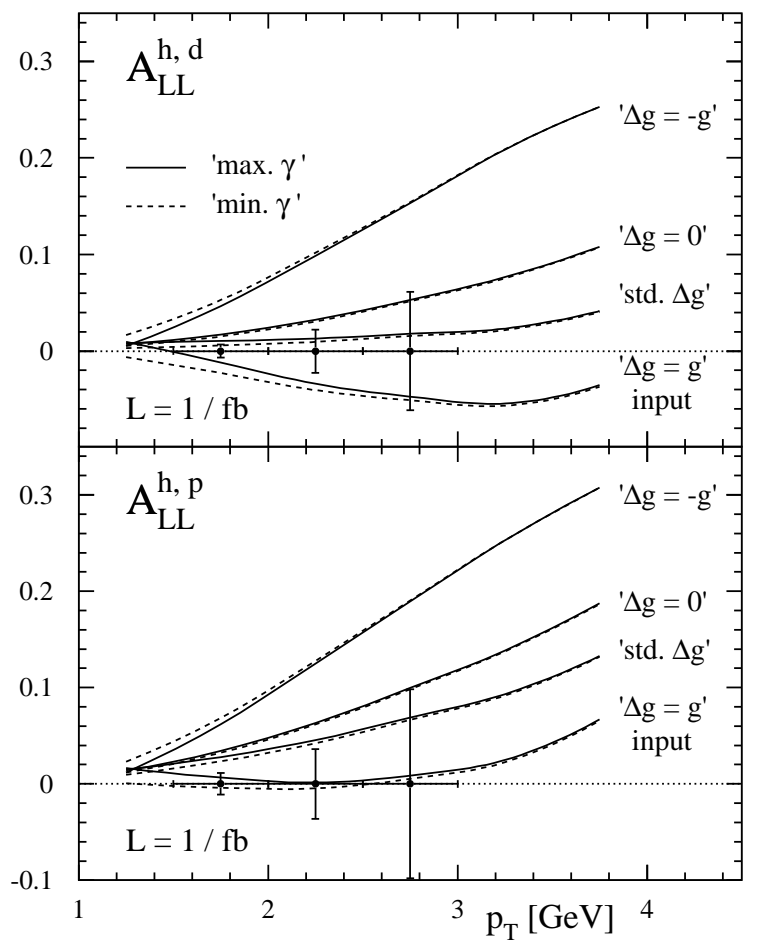

Fig. 7. As in Figs. 4 and 6 but now for the photoproduction of charged hadrons (see text).

described in Figs. 5 (a) and (b), but that the cancellation between the $\gamma g$ and $\gamma q$ channels is less complete such that the resolved cross section is somewhat less relevant here.

So far we have only considered the production of neutral pions where fragmentation functions were shown to work reasonably well also at rather low scales 26. However, the sum of charged hadrons, predominantly pions, but also kaons and protons, is equally important exper- 


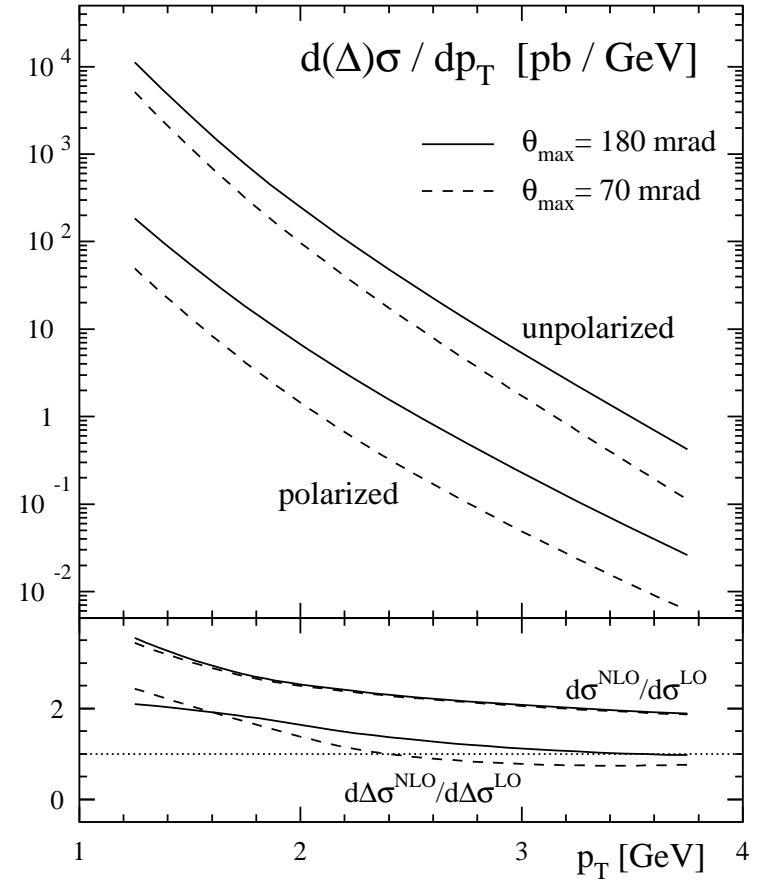

Fig. 8. Unpolarized and polarized $p_{T}$-differential cross sections at NLO for the reaction $\mu d \rightarrow \mu^{\prime} \pi^{0} X$ for two different experimental setups: $\theta_{\max }=180 \mathrm{mrad}$ (solid) and, as in Fig. 2 $\theta_{\max }=70 \mathrm{mrad}$ (dashed). The lower panel shows the corresponding ratios of NLO and LO results ( $K$-factor).

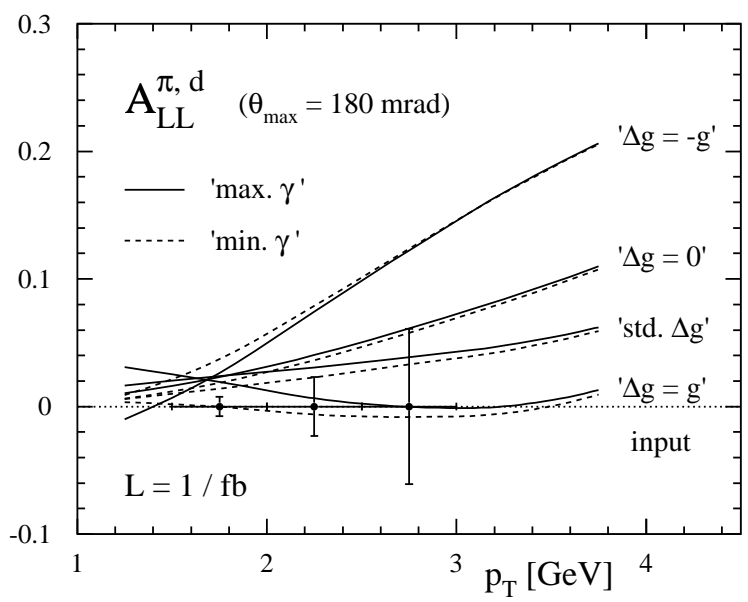

Fig. 9. As in Fig. 4 but now for $\theta_{\max }=180 \mathrm{mrad}$.

imentally as these are often more easily identified than neutral pions. This is also the case for Compass at the moment. In Fig. 7 we therefore present the relevant spin asymmetries $A_{\mathrm{LL}}^{h, d}$ and $A_{\mathrm{LL}}^{h, p}$ for deuteron and proton targets, respectively, for the reaction $\mu d(p) \rightarrow \mu^{\prime} h X$, where $h$ represents the sum of charged hadrons (pions, kaons, and protons). The results are obtained by employing the appropriate set of fragmentation functions, $D_{c}^{h^{+}+h^{-}}$of Ref. [25]. Not unexpectedly, the gross features of the spin asymmetries in charged hadron production are the same as in neutral pion production. Due to the larger rate for the sum of charged hadrons, the statistical precision is noticeably better than for $A_{\mathrm{LL}}^{\pi, d}$ which makes such a measurement potentially more interesting.

From our results for the spin asymmetries shown in Figs. 4, 6, and 7 it is obvious that a major difficulty in extracting the gluon polarization $\Delta g$ at fixed-target energies lies in the poor statistical accuracy at large $p_{T}$-values. This, however, is the region where perturbation theory is expected to be more reliable and where the uncertainties associated with the resolved photon contributions to the cross section are much better under control. It is therefore interesting to study whether the planned upgrade of the Compass experimental setup, which would lead to a much larger acceptance of $\theta_{\max }=180 \mathrm{mrad}$ and hence larger cross sections, could help.

In Fig. 8] we compare the polarized and unpolarized neutral pion photoproduction cross sections for the present setup with $\theta_{\max }=70 \mathrm{mrad}$ and for the proposed upgrade with $\theta_{\max }=180 \mathrm{mrad}$. The gain in cross section is a factor $4 \div 5$ depending on the $p_{T}$-value considered, yielding an improvement of the statistical accuracies for spin asymmetry measurements by about a factor of two. The lower panel of Fig. 8 shows that the respective $K$-factors do not change significantly when going from $\theta_{\max }=70 \mathrm{mrad}$ to $\theta_{\max }=180 \mathrm{mrad}$. However, not only does an increased angular acceptance improve the statistical accuracy, it also changes the interplay of direct and resolved subprocesses. It turns out that differences between the "maximal" and "minimal" $\Delta f^{\gamma}$ scenarios are more pronounced and persist towards larger $p_{T}$. This can be inferred from our estimate for the spin asymmetry $A_{\mathrm{LL}}^{\pi, d}$ shown in Fig. 9 if compared

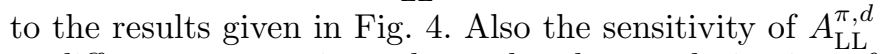
to different assumptions about the gluon polarization of the nucleon is somewhat smaller than before.

\subsection{Single-Inclusive Hadron Production at HERMES}

Single-inclusive hadron photoproduction can be also studied with the HERMES experiment at DESY where an electron (or positron) beam with $E_{e} \simeq 27.5 \mathrm{GeV}$ is scattered off a proton or deuterium gas target. The available c.m.s. energy of about $\sqrt{S} \simeq 7.5 \mathrm{GeV}$ is lower than at CompAss which even further limits the range of accessible transverse momenta. On average the electron beam polarization is $\mathcal{P}_{e} \simeq 53 \%$. For the polarization of the gas target we take $\mathcal{P}_{d} \approx \mathcal{P}_{p} \simeq 85 \%$, and, contrary to a solid-state target, there is no dilution, i.e., $\mathcal{F}_{p}=\mathcal{F}_{d}=1$. To estimate the statistical accuracies for a measurement of $A_{\mathrm{LL}}^{\pi, p}$ and $A_{\mathrm{LL}}^{\pi, d}$ below, we assume an integrated luminosity $\mathcal{L}$ of $50 \mathrm{pb}^{-1}$ and $200 \mathrm{pb}^{-1}$ for proton and deuterium targets, respectively. These numbers are based on the actual data already collected with the HERMEs experiment which is now running with transverse polarization most likely until the end of their experimental program. 


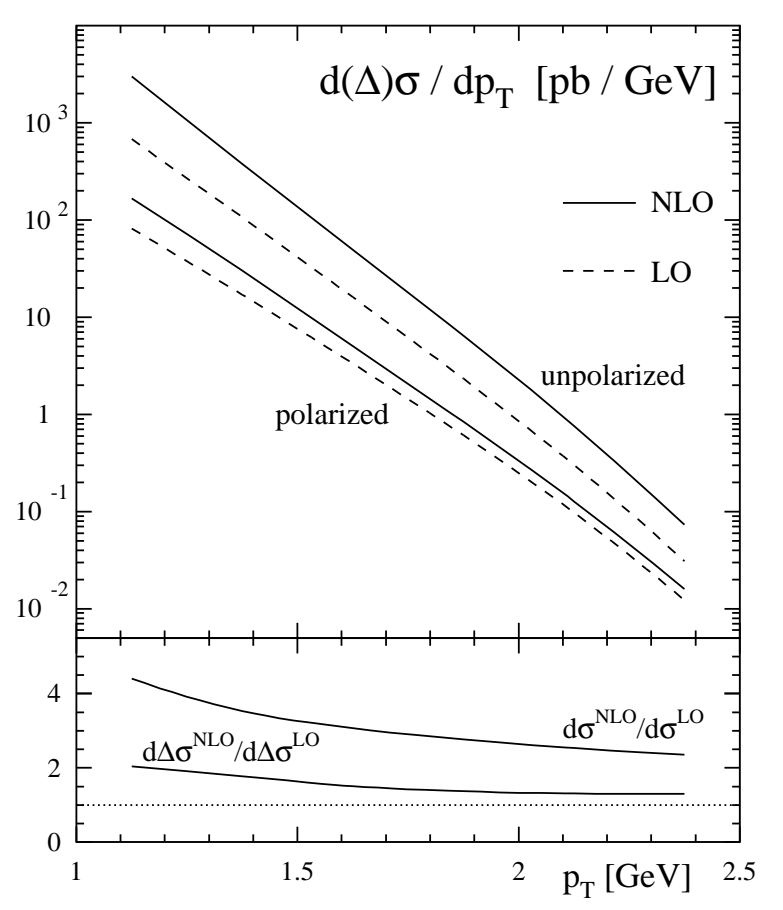

Fig. 10. Unpolarized and polarized $p_{T}$-differential singleinclusive cross sections at LO (dashed) and NLO (solid) for photoproduction of neutral pions, $e p \rightarrow e^{\prime} \pi^{0} X$, at $\sqrt{S}=$ $7.5 \mathrm{GeV}$, integrated over the angular acceptance of HERMES. The lower panel shows the ratios of NLO to LO contributions ( $K$-factor).

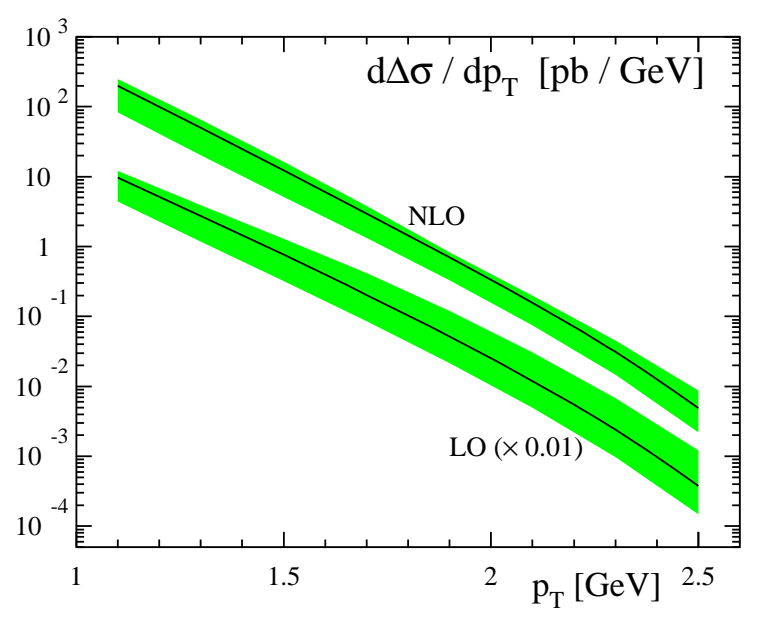

Fig. 11. As in Fig. 3 (b) but now for $e p \rightarrow e^{\prime} \pi^{0} X$ at Hermes.

The acceptance of the HERMES experiment allows the detection of pions in the angular range $40 \mathrm{mrad} \lesssim \theta \lesssim$ $220 \mathrm{mrad}$. The upper limit on $\theta$ corresponds to a minimal c.m.s. rapidity of $\eta_{c m s}^{\min } \simeq 0.2$. At small $p_{T}$ the lower limit on $\theta$, i.e., $\eta_{c m s}^{\max } \simeq 1.91$, sets a more stringent bound on $\eta_{c m s}^{\max }$ than purely kinematical considerations, $\eta_{c m s}^{\max }=$ $\cosh ^{-1}\left(\sqrt{S} / 2 p_{T}\right)$. For all our numerical studies we inte-

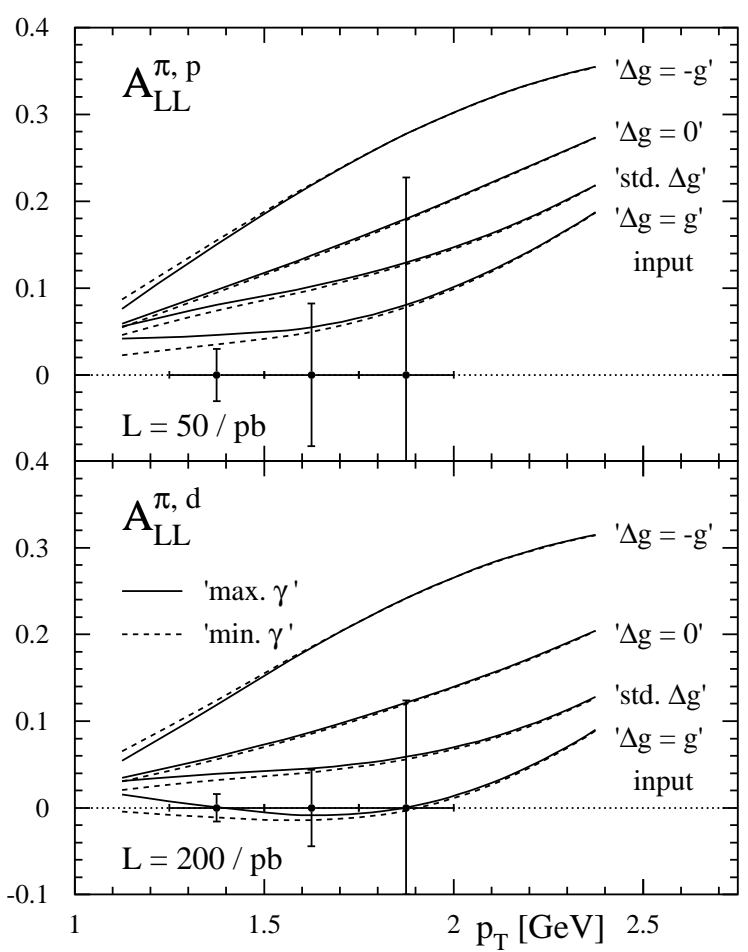

Fig. 12. Double-spin asymmetries $A_{\mathrm{LL}}^{\pi, p}$ and $A_{\mathrm{LL}}^{\pi, d}$ for HeRmeS kinematics at NLO for different gluon polarizations in the nucleon and minimal and maximal saturation of the polarized photon densities, dashed and solid lines, respectively. The "error bars" indicate the estimated statistical uncertainty assuming an integrated luminosity of $50 \mathrm{pb}^{-1}$ and $200 \mathrm{pb}^{-1}$ for $A_{\mathrm{LL}}^{\pi, p}$ and $A_{\mathrm{LL}}^{\pi, d}$, respectively.

grate over rapidity as before. We select photoproduction by demanding that the virtuality squared of the photon is less than $Q_{\max }^{2}=0.01 \mathrm{GeV}^{2}$. The photon flux with photon energies limited to the range $0.2 \leq y \leq 0.9$ is again modeled by Eq. (6).

The resulting polarized and unpolarized $p_{T}$-differential cross sections and $K$-factors for photoproduction of neutral pions are depicted in Fig.10] We only show results for a HERMes setup with a proton target, i.e., $e p \rightarrow e^{\prime} \pi^{0} X$, as results for a deuterium target are qualitatively very similar. Due to the small c.m.s. energy available the experimentally accessible transverse momenta $p_{T}$ are limited to a region where a successful and reliable application of perturbative methods cannot be taken for granted. This is emphasized, for instance, by the large value of the unpolarized $K$-factor which ranges from 2 to 4 . Even more dramatic are the uncertainties related to the choice for the renormalization and factorization scales which are shown for the polarized photoproduction cross section in Fig. [1] Again we have varied all scales simultaneously in the range $p_{T} / 2 \leq \mu_{r}=\mu_{f}=\mu_{f}^{\prime} \leq 2 p_{T}$. There is only a slight improvement when the NLO QCD corrections are taken into account. 


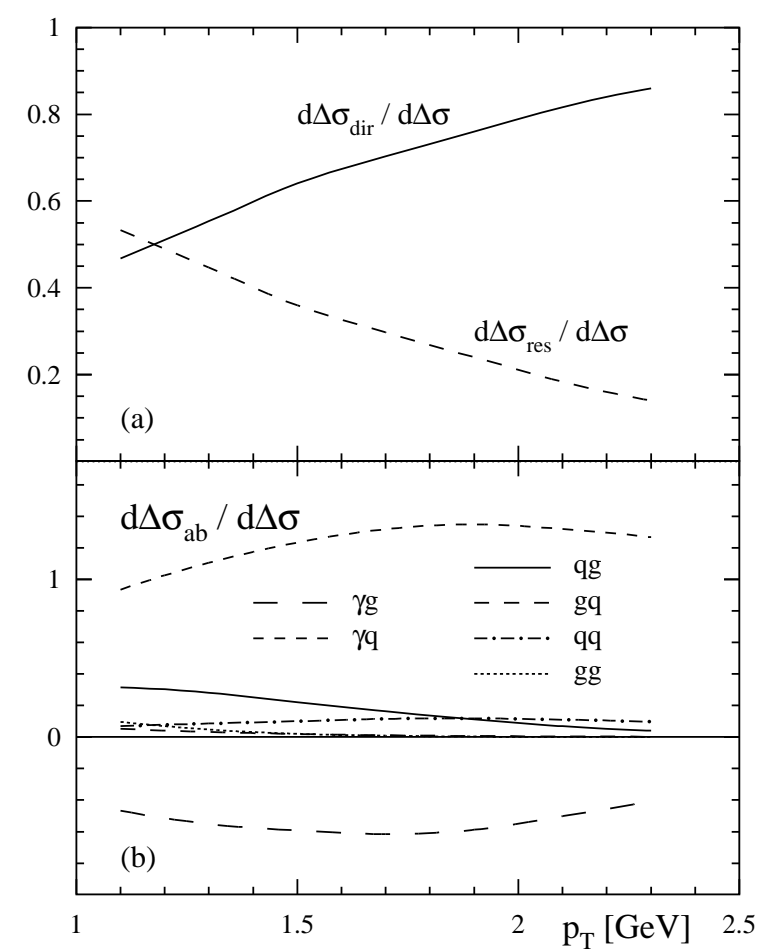

Fig. 13. (a) Direct and resolved $\mathrm{NLO}(\overline{\mathrm{MS}})$ and (b) $\mathrm{NLO}(\overline{\mathrm{MS}})$ contributions of different partonic channels $a+b \rightarrow c+X$ to the full NLO polarized photoproduction cross section shown in Fig. [10]

These results again clearly indicate that special care has to be taken in the interpretation of upcoming results in terms of pQCD and, ultimately, of $\Delta g$ at current fixedtarget experiments. It is perhaps even more important than at Compass energies that studies of the theoretically better predicted unpolarized cross section accompany studies with polarization. All reservations notwithstanding, we analyze in Fig. 12 the sensitivity of hadron photoproduction at HERMES to the gluon polarization of the nucleon. We show the double spin asymmetries $A_{\mathrm{LL}}^{\pi, p}$ and $A_{\mathrm{LL}}^{\pi, d}$ at NLO for different assumptions about the gluon polarization of the nucleon and the polarized parton densities of the photon. Again, we estimate the statistical error for such a measurement with the help of Eq. (11) with the parameters as specified at the beginning of this section. Clearly, meaningful results about $\Delta g$ can be only obtained for $p_{T} \approx 1.5 \mathrm{GeV}$. Due to the larger data sample available, results obtained with the deuteron target are much more discriminating than with the proton target.

Figure 12 also shows that the uncertainties from estimating the resolved contribution to the cross section are not very pronounced even at the lowest $p_{T}$-values shown. To examine the importance of the resolved contribution at HeRmes further, we show in Figs. 13 (a) and (b) the relative contributions of the direct and resolved and different partonic subprocesses $a+b \rightarrow c+X$ to the full photopro- duction cross section, respectively. It turns out that for a proton target the direct photon contribution $d \Delta \sigma_{\text {dir }}$ dominates in the entire $p_{T}$-range. Contrary to the situation for Compass, cf. Figs. [5 (a) and (b), the cancellation of the two direct channels $\gamma g$ and $\gamma q$ in the sum $d \Delta \sigma_{\text {dir }}$ is less complete. All resolved subprocesses are small and, for the same reasons as discussed before for CompAss, the bulk of $d \Delta \sigma_{\text {res }}$ stems from the scattering of a quark carrying a large momentum fraction of the photon off a gluon in the nucleon [solid line in Fig. 13 (b)]. For a deuteron target one obtains qualitatively similar results. However, at the small $p_{T}$-values shown the resolved contribution is more important due to a more pronounced cancellation of the $\gamma g$ and $\gamma q$ subprocesses in $d \Delta \sigma_{\mathrm{dir}}$.

\subsection{Comparison to the E155 Data}

As we mentioned in the introduction, there is already a data set on the spin asymmetry in single-inclusive hadron production by the E155 collaboration [16], and it is of course interesting to compare our NLO calculations to these data. The main data set of E155 is for $e N \rightarrow H^{ \pm} X$, where $H^{+}\left(H^{-}\right)$denotes any hadron of identified positive (negative) charge. Only for the data at the larger scattering angle $\theta=5.5^{\circ}$ are the observed hadron transverse momenta large enough for a sensible comparison to pQCD hard-scattering. Here we use again the various GRSV sets of polarized parton distributions, and the "maximal" set of polarized photon densities for the resolved contribution. We use the fragmentation functions of Ref. 30] which provide separate sets of $\mathrm{H}^{+}$and $\mathrm{H}^{-}$fragmentation functions. Finally, because the scattered electron was not observed for the E155 data, we use the Weizsäcker-Williams spectrum in Eq. (6) in the case when all photon virtualities are integrated, and we do not impose a cut on the variable $y$.

Figure 14 shows the comparison of our NLO calculations to the data of E155 for $H^{+}$and $H^{-}$production off proton and deuteron targets. One can see that it is difficult to achieve a quantitative overall agreement for a given set of GRSV polarized parton densities. On the other hand, the results show very large variation with the polarized parton distributions, and fine details of the densities will therefore matter. This is in particular so as there are again strong cancellations between the direct and resolved contributions, and even within these. One should also keep in mind that the uncertainty in the charge separation of the available sets of $H^{+}$and $H^{-}$fragmentation functions is currently very hard to quantify and certainly large. We finally note that there is also room for the "soft" non-perturbative contributions to the asymmetry discussed in [18, even though the data do not give compelling evidence for their presence either.

\section{Discussion and Conclusions}

To summarize, in this paper we have presented a detailed phenomenological study of single-inclusive hadron photo- 


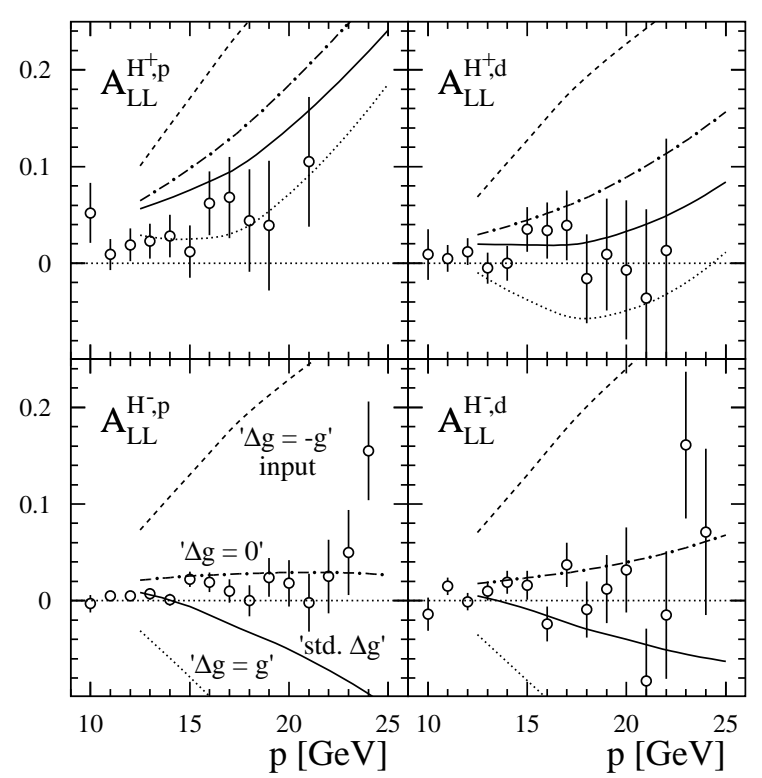

Fig. 14. Comparison of our NLO calculations to the E155 data for the double-spin asymmetries for $H^{+}$and $H^{-}$production in electron scattering off proton and deuteron targets at scattering angle $\theta=5.5^{\circ}$ [16]. In each of the four panels, different line styles distinguish the different GRSV sets of polarized parton distributions used in the calculation.

production at fixed-target energies with particular emphasis on the kinematics relevant for the Compass and HERMES experiments. We have also compared our calculations to already available data from the E155 experiment. All calculations were consistently performed at the next-to-leading order of perturbative QCD. We have carefully explored and critically discussed the applicability of the perturbative methods in the region of relatively low momentum transfers characterized by the $p_{T}$-values accessible in fixed-target experiments. Both the $K$-factors as well as uncertainties due to the choice of the renormalization and factorization scales turn out to be sizable; the latter only slightly improving when NLO corrections are taken into account.

All this indicates that the pQCD framework in the COMPASS and HeRmes kinematic regimes is delicate, and calls for a "reference measurement" of the unpolarized photoproduction cross section before the spin asymmetry can be analyzed in terms of $\Delta g$. If the unpolarized cross sections turn out to be in reasonable agreement with theory, measurements of the corresponding spin asymmetries by Compass and Hermes will add valuable information about the gluon polarization of the nucleon at momentum fractions $x \simeq 0.1 \div 0.3$. To access these large $x$-values at a polarized $p p$ collider like RHIC requires to detect finalstate hadrons, photons, or jets at rather high $p_{T}$ where the rate is small. The theoretical analysis of the unpolarized cross sections at COMPASS and HERMES may well require to pursue avenues like performing all-order resummations of large logarithms in the perturbative series.
Finally, it turned out that the resolved photon contribution to the cross section is sizable, and, under certain conditions, even dominant, but independent of the details of the non-perturbative spin structure of the polarized photon which is completely unknown so far. Analyzing the data with the approximation $d \Delta \sigma_{\text {res }} \approx 0$ would lead to incorrect conclusions about $\Delta g$. To access the parton content of polarized photons certainly requires a future polarized lepton-hadron collider facility such as the eRHIC project at BNL.

\section{Acknowledgments}

We are grateful to E. Aschenauer, P. Liebing, and V. Mexner (Hermes), to J. Friedrich, R. Kuhn, J.-M. Le Goff, G. Mallot, S. Paul, and J. Pretz (Compass), and to P. Bosted (E155) for valuable discussions and information about the experimental details of their photoproduction measurements. We also thank A. Afanasev for drawing our attention to the E155 data and to his earlier work. W.V. is grateful to RIKEN, Brookhaven National Laboratory and the U.S. Department of Energy (contract number DE-AC02-98CH10886) for providing the facilities essential for the completion of this work. B.J. would like to thank the Sonderforschungsbereich TR9 of the Deutsche Forschungsgemeinschaft for support.

\section{References}

1. See, for example: G. Bunce, N. Saito, J. Soffer, and W. Vogelsang, Annu. Rev. Nucl. Part. Sci. 50, 525 (2000).

2. Compass Collaboration, G. Baum et al., CERN/SPSLC 9614 (1996).

3. Hermes Collaboration, "The Hermes Physics Program \& Plans for 2001-2006", DESY-PRC (2000).

4. A. Bravar, D. von Harrach, and A. Kotzinian, Phys. Lett. B421, 349 (1998); A. Airapetian et al., Hermes Collaboration, Phys. Rev. Lett. 84, 2584 (2000); C. Bernet, Compass Collaboration, hep-ex/0405073 B. Adeva et al., Spin Muon Collaboration, Phys. Rev. D70, 012002 (2004).

5. S.B. Libby and G. Sterman, Phys. Rev. D18, 3252 (1978); R.K. Ellis, H. Georgi, M. Machacek, H.D. Politzer, and G.G. Ross, Phys. Lett. 78B, 281 (1978); Nucl. Phys. B152, 285 (1979); D. Amati, R. Petronzio, and G. Veneziano, Nucl. Phys. B140, 54 (1980); Nucl. Phys. B146, 29 (1978); G. Curci, W. Furmanski, and R. Petronzio, Nucl. Phys. B175, 27 (1980); J.C. Collins, D.E. Soper, and G. Sterman, Phys. Lett. B134, 263 (1984); Nucl. Phys. B261, 104 (1985); J.C. Collins, Nucl. Phys. B394, 169 (1993).

6. S.S. Adler et al., Phenix Collaboration, Phys. Rev. Lett. 91, 241803 (2003); J. Adams et al., STAR Collaboration, Phys. Rev. Lett. 92, 171801 (2004); G. Rakness, Star Collaboration, nucl-ex/0501026

7. K. Okada, Phenix Collaboration, hep-ex/0501066

8. B. Jäger, A. Schäfer, M. Stratmann, and W. Vogelsang, Phys. Rev. D67, 054005 (2003).

9. F. Aversa, P. Chiappetta, M. Greco, and J.-Ph. Guillet, Nucl. Phys. B327, 105 (1989); D. de Florian, Phys. Rev. D67, 054004 (2003). 
10. P. Aurenche et al., Phys. Lett. B140, 87 (1984); Nucl. Phys. B297, 661 (1988); H. Bear et al., Phys. Rev. D42, 61 (1990); Phys. Lett. B234, 127 (1990); L.E. Gordon and W. Vogelsang, Phys. Rev. D48, 3136 (1993); D50, 1901 (1994).

11. S.S. Adler et al., Phenix Collaboration, Phys. Rev. Lett. 93, 202002 (2004); Y. Fukao, PHEnIx Collaboration, hep-ex/0501049

12. B. Jäger, M. Stratmann, S. Kretzer, and W. Vogelsang, Phys. Rev. Lett. 92, 121803 (2004).

13. B. Jäger, M. Stratmann, and W. Vogelsang, Phys. Rev. D68, 114018 (2003).

14. See http://www.bnl.gov/eic for information concerning the eRHIC/EIC project, including the "Whitepaper" BNL68933.

15. A recent review can be found, e.g., in: M. Klasen, Rev. Mod. Phys. 74, 1221 (2002).

16. P. L. Anthony et al., E155 Collaboration, Phys. Lett. B458, 536 (1999).

17. A. Afanasev, C.E. Carlson, and C. Wahlquist, Phys. Lett. B 398, 393 (1997); Phys. Rev. D58, 054007 (1998).

18. A. Afanasev, C.E. Carlson, and C. Wahlquist, Phys. Rev. D61, 034014 (2000).

19. L. Apanasevich et al., E706 Collaboration, Phys. Rev. Lett. 81, 2642 (1998); P. Aurenche, M. Fontannaz, J. P. Guillet, B. A. Kniehl, and M. Werlen, Eur. Phys. J. C9, 107 (1999); ibid. 13, 347 (2000); U. Baur et al., hep-ph/0005226. C. Bourrely and J. Soffer, Eur. Phys. J. C36, 371 (2004).

20. D. de Florian and W. Vogelsang, Phys. Rev. D57, 4376 (1998).

21. D. de Florian and S. Frixione, Phys. Lett. B457, 236 (1999).

22. J. Pumplin et al., JHEP 0207, 012 (2002); H.-L. Lai et al., Eur. Phys. J. C12. 375 (2000).

23. M. Glück, E. Reya, and A. Vogt, Phys. Rev. D46, 1973 (1992).

24. M. Glück, E. Reya, M. Stratmann, and W. Vogelsang, Phys. Rev. D63, 094005 (2001).

25. B.A. Kniehl, G. Kramer, and B. Pötter, Nucl. Phys. B582, $514(2000)$.

26. E. Christova, S. Kretzer, and E. Leader, Eur. Phys. J. C22, 269 (2001).

27. M. Glück, E. Reya and C. Sieg, Phys. Lett. B 503, 285 (2001); Eur. Phys. J. C 20, 271 (2001).

28. M. Glück and W. Vogelsang, Z. Phys. C55, 353 (1992); ibid. C57, 309 (1993); M. Glück, M. Stratmann, and W. Vogelsang, Phys. Lett. B337, 373 (1994).

29. D. de Florian and W. Vogelsang, Phys. Rev. D71, 114004 (2005).

30. S. Kretzer, Phys. Rev. D62, 054001 (2000). 